\title{
CUANTIFICACIÓN ELECTROQUÍMICA DE MERCURIO (II) EN MEDIO ACUOSO SOBRE UN MICROELECTRODO DE FIBRA DE CARBONO MODIFICADO CON NANOPARTÍCULAS DE ORO
}

\section{ELECTROCHEMICAL QUANTIFICATION OF MERCURY (II) IN AQUEOUS MEDIUM ON CARBON FIBER MICROELECTRODE MODIFIED WITH GOLD NANOPARTICLES}

\section{Nicole Pérez-Uresta ${ }^{1}$, Diego Bolaños-Mendez ${ }^{1}$, Marjorie Montero-Jiménez ${ }^{1}$, Lenys Fernández ${ }^{1 *}$, Alexis Debut ${ }^{2}$, Patricio Espinoza-Montero ${ }^{1 *}$}

Recibido: 15 de mayo 2020 / Aceptado: 20 de noviembre 2020 DOI: 10.26807/ia.v9i1.167

Palabras clave: Fibra de carbono, Mercurio, Nanopartículas de oro, Voltamperometría de pulso diferencial. Keywords: Carbon fiber, Differential pulse voltammetry, Gold nanoparticles, Mercury.

1 Pontificia Universidad Católica del Ecuador, Escuela de Ciencias Químicas, Quito-Ecuador (pereznicole2a@hotmail.com, diegogeovanny222@hotmail.es, marjorie_cpp@ hotmail.com, *correspondencia: Imfernandez@puce.edu.ec,pespinoza646@puce.edu.ec)

2 Universidad de las Fuerzas Armadas ESPE, Centro de Nanociencia y Nanotecnología, SangolquíEcuador (apdebut@espe.edu.ec) 


\section{RESUMEN}

El mercurio es considerado un contaminante altamente peligroso debido a su elevada toxicidad y a su carácter acumulativo. Presenta varios efectos en la salud humana, tales como: trastornos del sistema nervioso, deterioro intelectual, problemas gastrointestinales y cáncer. El uso de técnicas electroquímicas es una alternativa para la cuantificación de mercurio a niveles traza, debido a su bajo costo y buena sensibilidad. En este trabajo de investigación se evaluó un microelectrodo de fibra de carbono modificado con nanopartículas de oro para la determinación de mercurio (II) en medio acuoso. Se realizó la modificación superficial de la fibra de carbono electrodepositando nanopartículas de oro, a un potencial de $-0,1 \mathrm{~V}$ durante 100 s, y se utilizó microscopía electrónica de barrido y voltamperometría cíclica para confirmar la presencia de nanopartículas de oro. Se cuantificó el mercurio (II) mediante voltamperometría de redisolución anódica de pulso diferencial. Se obtuvo un rango lineal, en la curva de calibración, entre 50 - $100 \mu \mathrm{g} \mathrm{L}^{-1}$ de mercurio (II). Los límites de detección y de cuantificación obtenidos fueron de 38 y $46 \mu \mathrm{g} \mathrm{L}^{-1}$, respectivamente. La validación de la metodología se realizó mediante porcentaje de recuperación, obteniendo valores entre 94 y 104 \% y desviación estándar relativa (RSD, por sus siglas en inglés) entre 3,6 y 4,7 \%. El método propuesto muestra características útiles para su implementación en el monitoreo de contaminación por mercurio.

\section{ABSTRACT}

Mercury is considered a highly dangerous pollutant due to its high toxicity and its cumulative nature. It has several effects on human health, such as: nervous system disorders, intellectual disorders, gastrointestinal problems and cancer. The use of electrochemical techniques is an alternative for the determination of mercury due to its low cost and good sensitivity. This research evaluated a carbon fiber microelectrode modified with gold nanoparticles for the quantification of Mercury (II) in aqueous medium. The modification of the carbon fiber was carried out by electrodepositing gold nanoparticles on the surface, impo- 
sing $-0.1 \mathrm{~V}$ for $100 \mathrm{~s}$. Scanning electron microscopy and cyclic voltammetry was used to confirm the presence of gold nanoparticles. The Mercury (II) signal was measured by differential pulse anodic stripping voltammetry (DPASV). In the calibration curve, a linear response was obtained in the range of 50-100 $\mu \mathrm{g} \mathrm{L}{ }^{-1}$. The detection and quantification limits obtained were 38 and $46 \mu \mathrm{g} \mathrm{L}^{-1}$, respectively. The validation of the methodology was performed using the recovery percentage, obtaining recovery between 94 and $104 \%$ and Relative Standard Deviation (RSD) between 3.6 and $4.7 \%$. The proposed method shows useful characteristics for monitoring mercury.

\section{INTRODUCCIÓN}

Los metales pesados se caracterizan por su toxicidad, ya que no son degradables y tienden a bioacumularse en animales, plantas y seres humanos. El mercurio es considerado uno de los metales con mayor impacto ambiental y su grado de toxicidad depende de la forma en la que se encuentre (elemental, orgánico e inorgánico). Este elemento puede acumularse en órganos y tejidos vitales como: corazón, hígado y cerebro, ocasionando trastornos en el sistema nervioso, cáncer, daño renal, deterioro intelectual e incluso puede llegar a causar la muerte (Abollino et al., 2008). Su presencia en el medio am- biente, se debe principalmente a emisiones de origen natural (liberación de gases de materiales geológicos) y emisiones de origen antropogénico (procesos industriales) (Martínez, 2004).

Las fuentes industriales, agrícolas, la escorrentía superficial y la deposición atmosférica (a través de las corrientes de aire), son las principales rutas de ingreso de mercurio al medio acuático. Los cuerpos de agua superficiales y sus sedimentos, son los medios donde ocurren los procesos de transformación del mercurio inorgánico $\left(\mathrm{Hg}^{2+}\right)$ a metilmercurio 
$\left(\mathrm{CH}_{3} \mathrm{Hg}^{+}\right)$por acción de bacterias. La forma orgánica se considera más tóxica, ya que tiende a difundirse, bioconcentrarse y bio-magnificarse fuertemente a lo largo de la cadena trófica del sistema marino. Desde su ingreso a través de la acumulación en algas, Ilegando a peces, y finalmente a algunos mamíferos e incluso a seres humanos (Badilla, 2018; Richter, Toral, \& Abbott, 2002).

Diversas organizaciones a nivel mundial han establecido límites máximos de concentración de mercurio, con el fin no solo de preservar la salud humana, sino también la integridad del medio ambiente (Sabino et al., 2015). En Ecuador, la Norma de Calidad Ambiental y Descarga de Efluentes: Recurso Agua, establece un límite de $0,001 \mathrm{mg} \mathrm{L}^{-1}$ para aguas de consumo y de uso agrícola; y 0,00018 $\mathrm{mg} \mathrm{L}^{-1}$ para aguas subterráneas; mientras que la Norma INEN 1108:2011 ha establecido un límite máximo de $0,006 \mathrm{~g} \mathrm{~L}^{-1}$ para el agua potable (INEN, 2014).

Se han reportado varios estudios para la determinación de mercurio empleando técnicas convencionales, como la espectrometría de absorción atómica de vapor frío, espectrometría de fluorescencia y espectrometría de masas con plasma acoplado inductivamente; sin embargo, las técnicas electroquímicas resultan una buena alternativa debido a su bajo costo, facilidad, rapidez y buena sensibilidad.

Diversos estudios han reportado, el uso de electrodos sólidos (disco de oro, película de oro, carbón vítreo) para el análisis de mercurio por diferentes técnicas voltamperométricas (Abollino et al., 2008; Bonfil et al., 2000). Actualmente, se propone la modificación química de la superficie de los electrodos con oro, para mejorar el efecto de preconcentración y la sensibilidad en la determinación de mercurio, ya que el oro se describe en la literatura como el material con mayor afinidad por este metal (Bernalte et al., 2011). Autores como Li et al. (2016), determinaron trazas de mercurio en agua mediante voltamperometría de pulso diferencial empleando un electrodo de oro, obteniendo recuperaciones del $81 \%$ al 107 \% y un límite de detección de 0,05 g L-1., Bernalte et al. (2012), por su parte, cuantificaron mercurio en muestras de agua mediante voltamperometría de redisolución anódica, 
utilizando electrodos de oro comerciales impresos con serigrafía. Otro ejemplo de un estudio similar es el reportado por Abollino et al. (2008), donde se investigaron las variables instrumentales involucradas en la determinación de $\mathrm{Hg}(\mathrm{II})$ en agua potable y muestras farmacéuticas, sobre un electrodo de oro.

Hasta el momento, en el Ecuador se tienen pocos reportes acerca del análisis de metales mediante técnicas electroquímicas, como voltamperometría de redisolución anódica de pulso diferencial, pese a su detección rápida, sensible y selectiva. Por otro lado, las fibras de carbono aparecen como un material prometedor para la elaboración de nanoelectrodos para la detección de metales como el mercurio, éste incluso podría ser mejorado en su característica electroactiva al cubrirlo con nanopartículas de oro (Carrera et al., 2017). Con base en los antecedentes mencionados, el objetivo de este trabajo fue utilizar un electrodo de fibra de carbono modificado con nanopartículas de oro para la determinación de $\mathrm{Hg}$ (II) en medio acuoso mediante voltamperometría de redisolución anódica de pulso diferencial.

\section{MATERIALES Y MÉTODOS}

\section{Reactivos}

Ácido clorhídrico ( $\mathrm{HCl}$ ) libre de metales (37\% p/p, Merk), ácido sulfúrico $\left(\mathrm{H}_{2} \mathrm{SO}_{4}\right)(97,00 \% \mathrm{p} / \mathrm{p}$ Merck), estándar de mercurio $(\mathrm{Hg}(\mathrm{II}))$ grado absorción atómica (1000 mg L-1, AccuStandard), estándar de oro (Au(III)) (995 mg/L, Spectrum), metanol anhidro (99,80\% Sigma-Aldrich) y agua destilada/desionizada, resistividad de $18 \mathrm{M} \Omega$. Para cada ensayo electroquímico se desgasificó la solución bur- bujeando nitrógeno 99,99 \% durante 5 minutos.

\section{Instrumentación}

Los experimentos se llevaron a cabo utilizando un sistema de tres electrodos conformado por: un electrodo de fibra de carbono PANEX 35 de Zoltek modificado con nanopartículas de oro como electrodo de trabajo, una barra 2B de grafito marca STAEDTLER como contraelectrodo y un elec- 
trodo de referencia de $\mathrm{Ag} / \mathrm{AgCl}$. El potenciostato $\mathrm{CH}$ Instruments (modelo CHI1230C, serie F1603) se utilizó para la obtención de voltammogramas y espectros.

El análisis de microscopía electrónica de barrido (SEM) se realizó empleando un microscopio electrónico de barrido marca Tescan modelo Mira3.

\section{Construcción del microelectrodo de fibra de carbono}

La fibra de carbono de aproximadamente $11 \mu \mathrm{m}$ de diámetro fue sometida a enjuagues sucesivos empleando las siguientes soluciones: ácido nítrico al $5 \%$, metanol, acetona y agua desionizada; posteriormente, se dejó secar a $40^{\circ} \mathrm{C}$ por 1 hora. La fibra se introdujo dentro de un tubo de polietileno de baja densidad (5 mm), obteniendo así una punta capilar moldeada por acción de calor, lo cual le dio al tubo una forma semejante a una pipeta. Posteriormente, la fibra se conectó a un cable de cobre eléctrico blindado usando una pintura conductora de plata y luego se colocó a la punta del tubo una gota de silicio caliente, permitiendo un se-
Ilado adecuado al solidificarse (Figura 1). Finalmente, se realizó un lavado con acetona y se dejó secar al ambiente (Carrera et al., 2017).

\section{Modificación del electrodo de tra-} bajo

Para la deposición de nanopartículas de oro sobre la superficie de la fibra de carbono, se sumergió la fibra dentro de una solución de $\mathrm{HCl}$ 0,5 mol $\mathrm{L}^{-1}$ que contenía $10 \mathrm{mg} \mathrm{L}^{-1}$ de un estándar de Au. Se aplicó un potencial contante de -0,1 V durante 60, 100 y 120 s (Berrazueta, 2015).

\section{Caracterización y activación del electrodo}

Se realizó la caracterización estructural colocando la fibra de carbono limpia y seca en un porta muestras y se la analizó en el SEM a un voltaje de aceleración de $15 \mathrm{kV}$ en modo de alto vacío.

Se evaluó la respuesta electroquímica del electrodo de trabajo en dos etapas: antes de la electrodeposición de las nanopartículas de $\mathrm{Au}$, y después de su modificación, empleando la técnica de voltamperometría cíclica 
(VC) en una solución 0,05 mol L-1 de $\mathrm{H}_{2} \mathrm{SO}_{4}$. La ventana de trabajo fue de $-0,20 \mathrm{a}+1,35 \mathrm{~V}$ a una velocidad de barrido de $100 \mathrm{mV} \mathrm{s}^{-1}$. Para la activación del electrodo modificado con nanopartículas de Au, se utilizó una solución de $\mathrm{HCl}$ 0,15 $\mathrm{mol} \mathrm{L}^{-1}$, aplicando dos ciclos de voltamperometría cíclica en las mismas condiciones de trabajo mencionadas anteriormente (Figura 1).

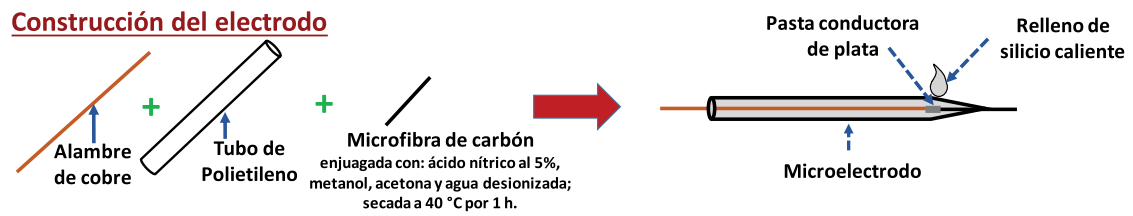

\section{Detección de $\mathrm{Hg}($ III)}
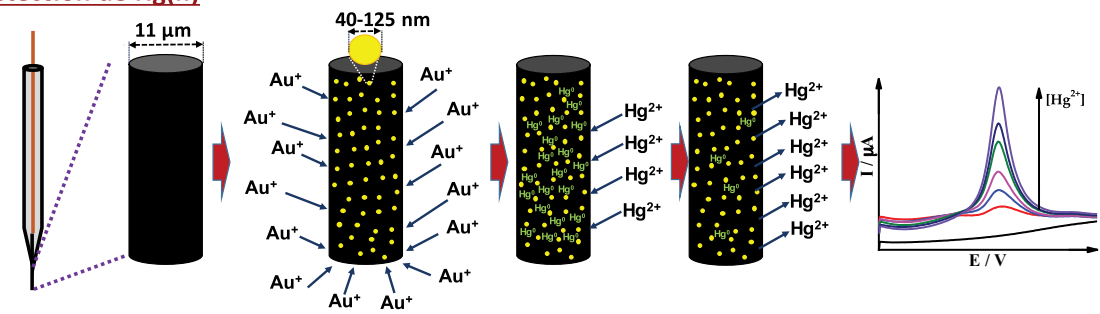

Microelectrodo-Fibra de carbón $\underset{\text { oro electrodepositadas }}{\longrightarrow}$ Nanopartículas de-
-

Preconcentración $\longrightarrow$ Redisolución de $\mathrm{Hg}$ (II) de $\mathrm{Hg}^{0}$ a $\mathrm{Hg}$ (II)

$\rightarrow$ Detección mediante DPASV

Figura 1. Construcción del microelectrodo de fibra de carbono y detección de $\mathrm{Hg}$ (II) por voltametría de redisolución anódica de pulso diferencial, adaptado de Carrera et al. (2017).

\section{Curva de calibración}

Se construyó una curva de calibración de 50, 60, 70, 80, 90 y $100 \mu \mathrm{g}$ $\mathrm{L}^{-1}$, a partir de una disolución patrón de $1000 \mathrm{mg} \mathrm{L}^{-1}$ de $\mathrm{Hg}$ (II). Como electrolito de soporte se empleó una disolución de $\mathrm{HCl} \mathrm{0,15} \mathrm{mol} \mathrm{L}^{-1}$, misma que se utilizó como blanco. Se aplicó la técnica de voltamperometría de redisolución anódica de pulso diferencial (DPASV, por sus siglas en inglés), en una ventana de trabajo de $-0,2$ a $0,3 \mathrm{~V}$, con un potencial de preacondicionamiento de $-0,8 \mathrm{~V}$ durante 60 segundos (Figura 1). 


\section{Figuras de mérito}

Para el cálculo de los límites de detección (LD) y cuantificación (LC), se utilizó la estimación de la mínima señal detectable descrita en la Guía de la EURACHEM (Magnusson \& Örnemark, 2014; Alonso et al., 2009) realizado a partir de 10 mediciones en el electrolito (solución del blanco) con el electrodo modificado. Se determinó la señal correspondiente a LD empleando la ecuación 1 y LC empleando la ecuación 2. El valor de estas señales se interpoló en la curva de calibración.

$$
\begin{gathered}
\mathrm{LDi} / \mathrm{A}=\mathrm{X}+3 \mathrm{~S} \\
\mathrm{LCi} / \mathrm{A}=\mathrm{X}+10 \mathrm{~S}
\end{gathered}
$$

Donde:

X: señal promedio de los blancos

S: desviación estándar de los blancos

Para la determinación de exactitud y precisión se realizaron dos medidas por día (repetibilidad) de dos soluciones preparadas a partir del estándar de mercurio (II) $\left(50 \mu \mathrm{g} \mathrm{L}^{-1}\right.$ y $\left.80 \mu \mathrm{g} \mathrm{L}^{-1}\right)$ en tres días diferentes (reproducibilidad).

\section{Cuantificación de $\mathrm{Hg}$ (II) en muestra de agua contaminada}

Se utilizó una muestra de agua de consumo (embotellada) a la cual se le fortificó con $65 \mu \mathrm{g} \mathrm{L}^{-1}$ de la solución estándar de $\mathrm{Hg}$ (II). Se llevó a cabo el método de adición estándar para la construcción de la curva de calibración. En la celda, se colocó 5 $\mathrm{mL}$ de la muestra fortificada junto con $5 \mathrm{~mL}$ de $\mathrm{HCl} 0,3 \mathrm{~mol} \mathrm{~L}^{-1}$ y se midió con DPASV. Después se añadió a la misma celda $50 \mu \mathrm{L}$ de una solución de Hg(II) de $1000 \mu \mathrm{g} \mathrm{L}^{-1}$ y se aplicó nuevamente DPASV. Se repitió este último procedimiento cinco veces más (Bader, 1980; Berrazueta, 2015; Tafur et al., 2018). La cantidad de Hg(II) se comprobó empleando la ecuación 3.

$$
\mathrm{CM}=\mathrm{b} / \mathrm{m} \times \mathrm{Cs} / \mathrm{Vm}
$$

Donde:

CM: concentración de la muestra b: ordenada al origen

Cs: concentración del estándar $\mathrm{m}$ : pendiente $\mathrm{Vm}$ : volumen de muestra 


\section{RESULTADOS}

Respuesta electroquímica de la microfibra de carbono modificada

La Figura 2 muestra la respuesta de la microfibra de carbono modificada con nanopartículas de oro frente a una muestra de $80 \mu \mathrm{g} \mathrm{L}^{-1}$ de $\mathrm{Hg}(\mathrm{II})$, mediante DPASV, a diferentes tiempos de preconcentración, utilizando un potencial constante de $0,1 \mathrm{~V}$.

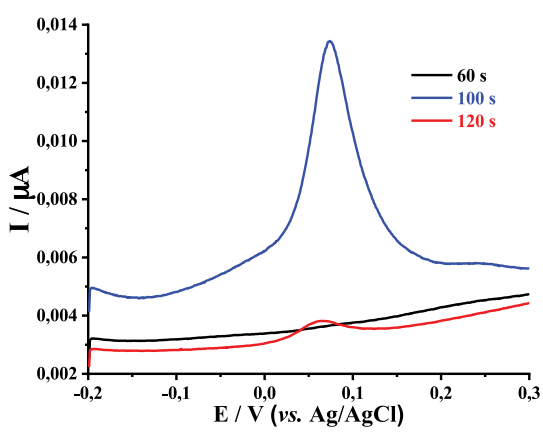

Figura 2. Detección de $80 \mu \mathrm{g} \mathrm{L}^{-1} \mathrm{Hg}(\mathrm{II})$, mediante DPASV, en la microfibra de carbono modificado con oro electrodepositado a diferentes tiempos

Caracterización del electrodo de microfibra de carbono modificado con nanopartículas de oro, mediante SEM.

La Figura 3 muestra las imágenes SEM a diferentes ampliaciones, Io cual permite evidenciar gracias al detector (BSE, por sus siglas en inglés) la presencia de las nanopartículas electrodepositadas en la superficie de la microfibra de carbono.

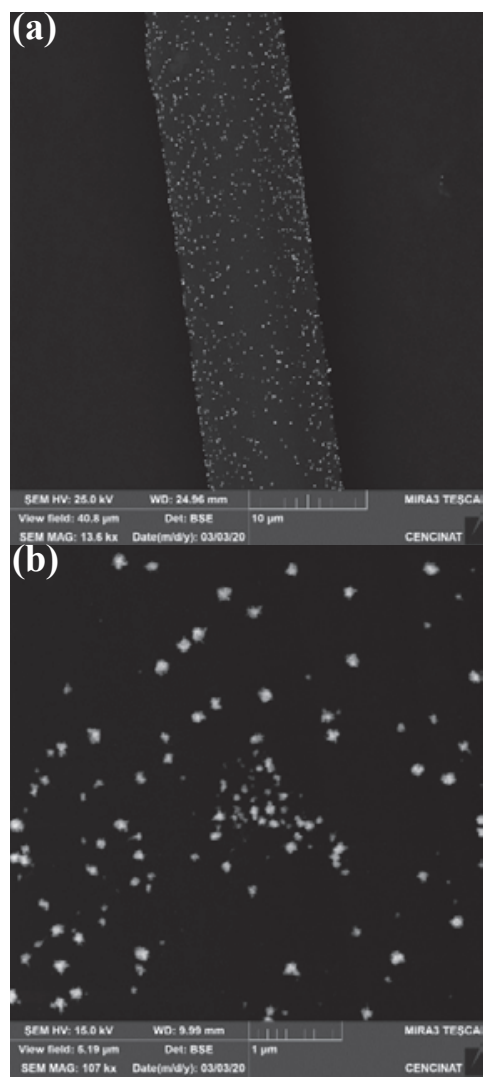

Figura 3. Imagen SEM de la microfibra de carbono modificada con nanopartículas de oro: (a) a 13,6 kx de ampliación, (b) a 107 kx 
Caracterización electroquímica mediante VC de la microfibra de carbono modificada con nanopartículas de oro

En la Figura 4 se muestra la respuesta electroquímica mediante VC de la microfibra de carbono sin modificar, y modificada con nanopartículas de oro en $\mathrm{H}_{2} \mathrm{SO}_{4}$ 0,5 mol L-1 a $100 \mathrm{mV}$ $\mathrm{s}^{-1}$.

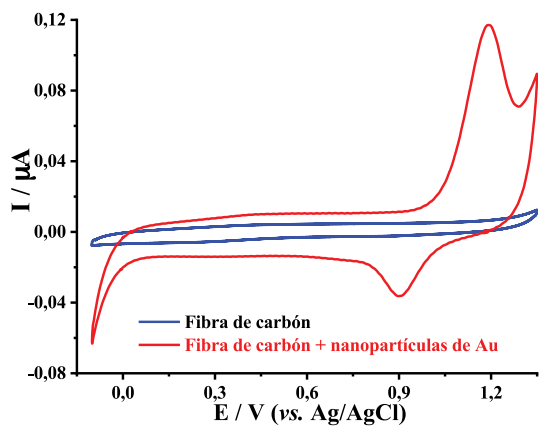

Figura 4. Voltamperograma de la microfibra de carbono sin modificar y modificada con nanopartículas de oro, en $0,05 \mathrm{~mol} \mathrm{~L}^{-1}$ de $\mathrm{H}_{2} \mathrm{SO}_{4}, \mathrm{a} \mathrm{v}=100 \mathrm{mV} \mathrm{s}^{-1}$

\section{Curva de calibración}

En la Figura 5 se muestran las señales obtenidas mediante DPASV para diferentes concentraciones de $\mathrm{Hg}(\mathrm{II})$, utilizando como electrodo de trabajo la microfibra de carbono modificado con nanopartículas de oro. En la Fi- gura 6 se ilustra la curva de calibración construida a partir de la Figura 5. Se tomó como referencia la intensidad de corriente máxima.

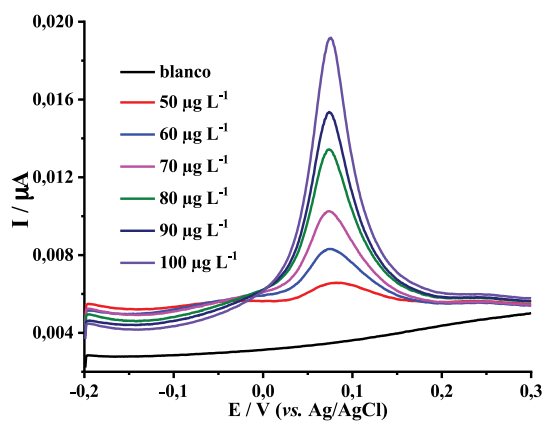

Figura 5. DPASV para diferentes concentraciones de $\mathrm{Hg}$ (II). A $10 \mathrm{mV} \mathrm{s}^{-1}, 0,8 \mathrm{~V}$ en la preconcentración de $\mathrm{Hg}$ y $-0,1 \mathrm{~V}$ de electrodeposición de oro

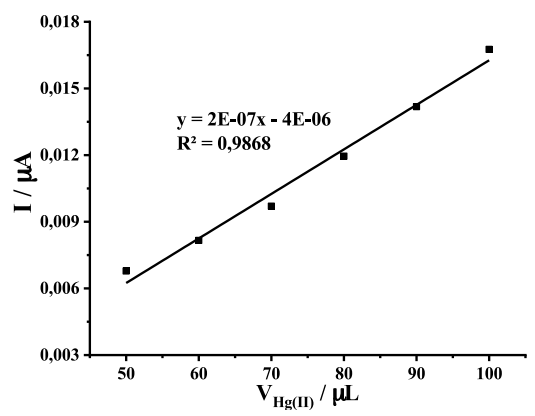

Figura 6. Curva de calibración lineal de $\mathrm{Hg}$ (II) correspondiente a las corrientes pico de las curvas de DPASV 


\section{Figuras de mérito}

El resultado obtenido de la estimación del límite de detección (LD) y límite de cuantificación (LC) se presenta en la Tabla 1.

Tabla 1. Límite de detección y Límite de cuantificación del método

\begin{tabular}{lcc}
\hline \multirow{2}{*}{ Compuesto } & \multicolumn{2}{c}{ Disolución de electrolito } \\
\cline { 2 - 3 } & $\begin{array}{c}\mathrm{LD} \\
\left(\mu \mathrm{L} \mathrm{L}^{-1}\right)\end{array}$ & $\begin{array}{c}\mathrm{LC} \\
\left(\mu \mathrm{g} \mathrm{L}^{-1}\right)\end{array}$ \\
\hline $\mathrm{Hg}(\mathrm{II})$ & 36 & 41 \\
\hline
\end{tabular}

Se determinó una recuperación media de $104 \%$ para la concentración de $50 \mu \mathrm{g} \mathrm{L}^{-1}$ y una recuperación media de 94 \% para la concentración de $80 \mu \mathrm{g} \mathrm{L}^{-1}$ con una desviación estándar relativa (RSD \%) de 3,6 y 4,0 respectivamente. En condiciones de repetibilidad, se determinó RSD (\%) entre 0,3 y $3,6 \%$; mientras que en condiciones de reproducibilidad se determinó RSD entre 3,7 y 4,7 \% (Tabla 2).

Tabla 2. Recuperaciones medias (\%) y RSD (\%) para soluciones de $\mathrm{Hg}(\mathrm{II})$

\begin{tabular}{lccc}
\hline \multirow{2}{*}{ Compuesto } & \multicolumn{3}{c}{ Disolución Acuosa } \\
\cline { 2 - 4 } & $\begin{array}{c}\text { Conc. } \\
\left(\mu \mathrm{g} \mathrm{L}^{-1}\right)\end{array}$ & $\begin{array}{c}\mathrm{R} \\
(\%)\end{array}$ & $\begin{array}{c}\text { RSD } \\
(\%)\end{array}$ \\
\hline $\mathrm{Hg}$ (II) & 50 & 104 & 3,6 \\
& 80 & 94 & 4,0 \\
\hline
\end{tabular}

\section{DISCUSIÓN}

Modificación del electrodo de trabajo

Durante la modificación del electrodo se realizaron algunas pruebas, variando el tiempo de electrodeposición de oro sobre la superficie de la fibra de carbono, con el fin de determinar el tiempo óptimo de deposición que permitiera obtener la cantidad de oro necesaria, y así lograr la mejor señal de mercurio en el rango de trabajo deseado. Para esto, en una solución de $\mathrm{HCl}$ 0,5 mol L-1 + $10 \mathrm{mg} \mathrm{L}^{-1}$ de $\mathrm{Au}(\mathrm{III})$, se realizó una cronoamperometría, donde se aplicó una diferencia de potencial constante en función del tiempo. Es durante este proceso, donde ocurre la reducción del $\mathrm{Au}$ (III) a $\mathrm{Au}^{0}$, lo cual induce la deposición de las nanopartículas de oro en la superficie del electrodo (Hezard et al., 2012). 
El tiempo óptimo determinado para que el oro se deposite sobre la superficie del electrodo ordenadamente en forma de nanopartículas fue de 100 $s$, ya que se obtuvo la señal de respuesta de corriente de detección de $\mathrm{Hg}$ (II) con mayor intensidad y mejor definición (Figura 2). Por otro lado, en tiempos inferiores a $100 \mathrm{~s}$, no se visualizó ningún pico y a un tiempo mayor se obtienen señales de corriente del mercurio poco definidas, lo cual podría ser asociado a una saturación de oro en la superficie de la microfibra de carbono.

\section{Caracterización del electrodo de mi- crofibra de carbono modificado con nanopartículas de oro}

La microscopía electrónica de barrido (SEM), se utilizó para confirmar la electrodeposición de las nanopartículas de oro sobre la superficie de la microfibra de carbono. En la Figura 3 se evidencia la presencia de las nanopartículas de oro en toda la superficie de la microfibra (las partes más brillosas); las cuales presentaron un tamaño entre 40-125 nm, de acuerdo con lo reportado en la literatura (Carrera et al., 2017; Berrazueta, 2015).
Adicionalmente, se observaron sitios en la superficie de la microfibra con aglomeraciones de las nanopartículas de oro, que pueden afectar la sensibilidad en las mediciones, debido a que esto corresponde a electrodepósitos poco ordenados. Sin embargo, se estima que su efecto insignificante debido a que estas aglomeraciones ocupan un área despreciable en comparación con el área total del electrodo (Carrera et al., 2017).

La caracterización por VC evidencia que el electrodo sin modificar (desnudo), no presenta picos en el voltamperograma en solución de $\mathrm{H}_{2} \mathrm{SO}_{4}$ (Figura 4), lo que indica que el electrodo está limpio y libre de interferencias. En contraste, el electrodo modificado con nanopartículas de oro presenta un incremento de corriente capacitiva, atribuido al aumento del área de superficie electroactiva por la presencia de la nanopartículas de oro (Carrera et al., 2017).

Los picos característicos de oxidación y reducción del oro en el electrodo modificado confirman la presencia de nanopartículas de oro depositadas en la superficie de la mi- 
crofibra. La señal de corriente a 1,2 $\mathrm{V}$, es característica de la formación de óxido de oro, de acuerdo con la reacción (1) (Carrera et al., 2017; Hezard et al., 2012):

$\mathrm{Au}+\mathrm{H}_{2} \mathrm{O} \rightleftarrows \mathrm{AuO}+2 \mathrm{H}^{+}+2 \mathrm{e}^{-}$

Por otro lado, el pico de reducción a $0,9 \mathrm{~V}$ se atribuye a la reducción de la capa de óxido formada en el barrido directo de potencial (Carrera et al., 2017; Hezard et al., 2012).

\section{Curva de calibración}

Las señales de la intensidad de corriente, Figura 5, son directamente proporcionales a concentración de $\mathrm{Hg}$ (II) en medio acuoso. La técnica empleada para la determinación de $\mathrm{Hg}$ (II) implica dos pasos: en primer lugar, el $\mathrm{Hg}(\mathrm{II})$ se preconcentra en la superficie del electrodo al aplicar un potencial de reducción (reacción 2), seguido de una etapa de redisolución (reacción 3) (Hezard et al., 2012) que tiene lugar al aplicar un barrido de potencial con la técnica de pulso diferencial en sentido oxidación (contrario al inicial) (Borja Vera, 2019; Carrera et al., 2017; Tafur et al., 2018). La señal de corriente de oxi- dación del mercurio aparece a 0,067 V.

$\mathrm{Hg}^{2+}+2 \mathrm{e}^{-} \rightleftarrows \mathrm{Hg}^{0}$

$\mathrm{Hg}^{0}+2 \mathrm{Cl}^{-} \rightleftarrows \downarrow \mathrm{HgCl}_{2}+2 \mathrm{e}^{-}$

Guan et al. (2018), obtuvieron resultados muy similares respecto a la sensibilidad del método al aplicar DPASV y usar un electrodo de tela de carbono modificado con nanopartículas de Au. En su estudio, las señales correspondientes a valores de concentración inferiores a $50 \mu \mathrm{g} \mathrm{L}^{-1}$ no eran distinguibles.

Al construir la curva de trabajo en el rango de concentración de $50 \mu \mathrm{g} \mathrm{L}^{-1}$ a $100 \mu \mathrm{g} \mathrm{L}^{-1}$ se obtuvo una linealidad con un coeficiente de correlación $\left(R^{2}\right)$ de 0,9868. Cada punto de la curva de calibración corresponde al valor medio obtenido de tres mediciones independientes de la corriente máxima de la señal.

\section{Figuras de mérito}

Estudios realizados previamente en condiciones similares (Bernalte et al., 2012; Guan et al., 2018), en los que se usó voltamperometría de onda cuadrada, reportan límites de cuanti- 
ficación más bajos $\left(0,6\right.$ y $\left.5 \mu \mathrm{g} \mathrm{L}^{-1}\right)$ que los obtenidos en este estudio. Estas diferencias se podrían atribuir al uso de electrodos con tamaño de partícula de oro menores, lo que puede dar una mayor sensibilidad y reproducibilidad en la detección. Adicionalmente, la limitación del método para la detección de $\mathrm{Hg}$ (II) en concentraciones bajas podría deberse a cambios estructurales de la superficie de la microfibra de carbono al realizar electrodeposiciones sucesivas de las nanopartículas de Au. Cambios en la estructura de la microfibra y la influencia de la electrodeposición de nanopartículas de oro sobre su superficie han sido poco estudiados y tiene alta incidencia en la robustez del método. De acuerdo con Guan et al. (2018), un inconveniente que puede presentarse durante el análisis, es la formación de amalgama Hg-Au que contamina la superficie del electrodo, principalmente a concentraciones altas, afectando la reproducibilidad del método (Guan et al., 2018).

Métodos instrumentales avanzados como la espectroscopía de absorción atómica de generación de hidruros, espectrometría de absorción atómica de horno de grafito y espectrometría de masas con plasma acoplado inductivamente (AAS-HG, AAS-GF e ICP-MS, por sus siglas en inglés) presentan límites de detección de: 0,009; 0,6 y 0,016 $\mu \mathrm{g} \mathrm{L}^{-1}$ respectivamente (Robinson et al., 2014). Estos límites son varios órdenes de magnitud inferiores en comparación con el reportado en nuestro método; sin embargo, es posible alcanzar valores más bajos realizando más estudios que permitan mejorar la señal de respuesta variando el potencial y tiempo de electrodeposición de nanopartículas de oro sobre la superficie de la fibra y ajustando las condiciones de DPASV. Cabe resaltar que la baja complejidad del método desarroIlado supondría una ventaja frente a los métodos instrumentales mencionados al ser capaz de realizar mediciones más rápidas e in situ a un bajo costo.

En cuanto a exactitud y precisión, Guan et al. (2018) obtuvieron recuperaciones medias entre 92,4 y 108,9 $\%$ y RSD (\%) entre 2,01 y $3,22 \%$ en dos matrices de agua distintas utilizando voltamperometría de redisolución anódica de onda cuadrada (SWSV, por sus siglas en inglés). AI 
aplicar el método establecido a una muestra de agua de consumo fortifi- cada con $65 \mu \mathrm{g} \mathrm{L}^{-1}$ de $\mathrm{Hg}$ (II), se obtuvo una recuperación de $104 \%$.

\section{CONCLUSIÓN}

Mediante voltamperometría cíclica se corroboró la presencia de nanopartículas de oro electrodepositadas sobre la superficie de las microfibras de carbono; los picos de oxidación y reducción del oro a 1,2 V y $0,9 \vee$ respectivamente. El desarrollo del electrodo permitió alcanzar límites de detección de $\mathrm{Hg}$ (II) de $36 \mu \mathrm{g} \mathrm{L}^{-1}$ y de cuantificación de $41 \mu \mathrm{g} \mathrm{\textrm {L } ^ { - 1 }}$ respectivamente, el rango lineal de trabajo se encontró entre 50-100 $\mu \mathrm{g} \mathrm{\textrm {L } ^ { - 1 }}$ de $\mathrm{Hg}(\mathrm{II})$, con un coeficiente de correlación de 0,987. La microfibra de carbono modificada con nanopartículas de oro resultó ser útil para la determinación de $\mathrm{Hg}(\mathrm{II})$ a partir de una concentración de $50 \mu \mathrm{g} \mathrm{L}{ }^{-1}$. El desempeño del método obtenido para el rango lineal estudiado, en cuanto a exactitud y precisión se considera aceptable, al ser comparable con el reportado en la literatura por varios autores. El método evaluado es confiable para la cuantificación de mercurio. Sin embargo, se sugiere realizar más estudios a profundidad, ya que es una forma barata de medir iones inorgánicos en medio acuoso.

AGRADECIMIENTOS: Los autores desean agradecer a la Ing. Karla Vizuete del Centro de Nanociencia y Nanotecnología, Universidad de las Fuerzas Armadas ESPE, por su soporte técnico en SEM. 


\section{LISTA DE REFERENCIAS}

Abollino, O., Giacomino, A., Malandrino, M., Piscionieri, G., \& Mentasti, E. (2008). Determination of mercury by anodic stripping voltammetry with a gold nanoparticle-modified glassy carbon electrode. Electroanalysis, 20(1), 75-83. https://doi.org/10.1002/elan.200704044

Bader, M. (1980). A systematic approach to standard addition methods in instrumental analysis. Journal of Chemical Education, 57(10), 703

Badilla Méndez, C. (2018). Evaluación del grado de contaminación con mercurio (Hg) en Laguna La Señoraza agua, sedimentos y biota. Universidad de Concepción. Facultad de Ciencias Ambientales.

Bernalte, E., Marín Sánchez, C., \& Pinilla Gil, E. (2012). Gold nanoparticles-modified screen-printed carbon electrodes for anodic stripping voltammetric determination of mercury in ambient water samples. Sensors and Actuators, B: Chemical, 161(1), 669-674. https://doi.org/10.1016/j.snb.2011.10.088

Bernalte, E., Sánchez, C. M., \& Gil, E. P. (2011). Determination of mercury in ambient water samples by anodic stripping voltammetry on screen-printed gold electrodes. Analytica Chimica Acta, 689(1), 60-64. https://doi.org/10.1016/j.aca.2011.01.042

Berrazueta Lanas, M. E. (2015). Desarrollo y validación de un método para la determinación de mercurio en aguas residuales, por voltamperometría (Master's thesis, Quito: UCE.).

Borja Vera, A. R. (2019). Determinación de Pb (II), Zn (II) en cervezas artesanales de Quito, mediante voltametría de redisolución anódica (Bachelor's thesis, PUCEQuito).

Carrera, P., Espinoza-Montero, P. J., Fernández, L., Romero, H., \& Alvarado, J. (2017). Electrochemical determination of arsenic in natural waters using carbon fiber ultramicroelectrodes modified with gold nanoparticles. Talanta, 166, 198-206. https://doi.org/10.1016/j.talanta.2017.01.056 
Guan, P., Guo, P. R., Liu, N., Zhang, F., \& Lei, Y. Q. (2018). The preparation of a flexible AuNP modified carbon cloth electrode and its application in electrochemical detection of $\mathrm{Hg}$ (ii) by continuous flow in environmental water. Analyst, 143(18), 4436-4441. https://doi.org/10.1039/c8an01284a

Hezard, T., Fajerwerg, K., Evrard, D., Collière, V., Behra, P., \& Gros, P. (2012). Influence of the gold nanoparticles electrodeposition method on $\mathrm{Hg}(\mathrm{II})$ trace electrochemical detection. Electrochimica Acta, 73(li), 15-22. https://doi.org/10.1016/j.electacta. 2011.10.101

INEN. (2014). Agua Potable. Requisitos. Nte Inen 1108. Retrieved from https://bibliotecapromocion.msp.gob.ec/greenstone/collect/promocin/index/assoc/HASH01a4.di r/doc.pdf\%0Ahttp://normaspdf.inen.gob.ec/pdf/nte/1108-5.pdf

Li, Z., Xia, S., Wang, J., Bian, C., \& Tong, J. (2016). Determination of trace mercury in water based on N-octylpyridinium ionic liquids preconcentration and stripping voltammetry. Journal of Hazardous Materials, 301, 206-213. https://doi.org/10. 1016/j.jhazmat.2015.08.061

Martínez, X. G. (2004). El mercurio como contaminante global: Desarrollo de metodologías para su determinación en suelos contaminados y estrategias para la reducción de su liberación al medio ambiente. Universitat Autònoma de Barcelona, 0, 246. https://doi.org/http://hdl.handle.net/10803/3174

Méndez, C. (2018). Evaluación del grado de contaminación con mercurio (Hg) en Laguna La Señoraza agua, sedimentos y biota (Doctoral dissertation, Universidad de Concepción. Facultad de Ciencias Ambientales)

Richter, P., Toral, M. I., \& Abbott, B. (2002). Anodic stripping voltammetric determination of mercury in water by using a new electrochemical flow through cell. Electroanalysis, 14(18), 1288-1293. https://doi.org/10.1002/1521-4109(200210)14:18< 1288::AID-ELAN1288>3.0.CO;2-5

Sabino, J. F. P., Valladares, B., Hernández, E., Oliva, B., Del Cid, M., \& Reyes, P. J. (2015). Determinación de arsénico y mercurio en agua superficial del lago de Atitlán. Ciencia, Tecnología y Salud, 2(2), 37-44. 
InfoANALÍTICA 9(1)

Enero 2021

Tafur, J., Espinoza-Montero, P., Manciati, C., Fierro-Naranjo, C., Swain, G. M., \& Fernández, L. (2018). Evaluation of BDD electrode in the determination of $\mathrm{Cd}(\mathrm{II})$, $\mathrm{Pb}$ (II) and $\mathrm{Hg}(\mathrm{II})$ in wastewater mining (Portovelo - Zaruma, Provincia de El Oro, Ecuador). Rev. Téc. Ing. Univ. Zulia, 41(2). Retrieved from http://produccioncientificaluz.org/index.php/tecnica/article/view/23484 